\title{
Factores que influyen en la motivación del deportista*
}

\author{
Diana Carolina Nope** \\ Jorge Luis Petro"** \\ Diego A. Bonilla
}

Recibido: octubre 30 del 2019 • Aceptado: febrero 19 del 2020

\section{Resumen}

En la actualidad, la motivación es uno de los campos de mayor interés en la práctica deportiva de alto rendimiento; de hecho, han surgido nuevos planteamientos y, al mismo tiempo, interrogantes acerca de las razones que llevan al deportista bien sea al incremento o bien al descenso de su rendimiento. El objetivo de este artículo es resumir diferentes factores que influyen sobre la motivación de los deportistas, para lo cual se llevó a cabo una revisión narrativa al analizar la literatura disponible en las bases de datos PubMed, Scielo y Dialnet que abordan este tema. Con un índice Price de $54 \%$, los artículos

Artículo de revisión. Financiación propia. Contribución de los autores: Idea original de la investigación, por Nope, D. C. Ejecución y redacción, por Nope D. C. y Bonilla D. A. Revisión y análisis, por Bonilla D. A. y Petro J. L. Adecuación a su versión final, por Bonilla D. A. Véase: https://www.redalyc.org/pdf/2351/235119246002.pdf

Citar como: Nope, C., Petro, J. y Bonilla, D. A. (2020). Factores que influyen en la motivación del deportista. Revista de investigación Cuerpo, Cultura y Movimiento, 10(2), 61-77. DOI: https://doi.org/10.15332/2422474x/6226

"* Psicóloga, Facultad de Psicología, Universidad Antonio Nariño, Cartago, Colombia. Correo electrónico: psicologa.carolinanopeocampo@gmail.com, ORCID: 0000-0002-4535-8554

\#** Doctorando en Ciencias Básicas Biomédicas, Universidad de Antioquia. Grupo de Investigación en Ciencias de la Actividad Física, el Deporte y la Salud (GICAFS), Universidad de Córdoba, Montería, Colombia. Correo electrónico: jorgelpetro@correo.unicordoba.edu.co, ORCID: 0000-0001-5678-1000

**** Doctorando en Biología Molecular y Biomedicina, Universidad del País Vasco. KDNA Genomics®, Universidad del País Vasco upv/EHu, Donostia-San Sebastián, España. Correo electrónico: dabonilla@dbss.pro, ORCID: 0000-0002-2634-1220 
revisados muestran que el estado de ánimo del deportista es de gran importancia, al considerar la influencia que este tiene sobre el desarrollo del autocontrol y la toma de decisiones, elementos fundamentales para la consecución de objetivos propuestos y el éxito deportivo. Este trabajo resalta la influencia que pueden tener diferentes factores sobre la motivación/desmotivación y el cumplimiento de los objetivos físico-deportivos, como los vínculos socioafectivos, el liderazgo y la cohesión del grupo, así como la participación del entrenador, la competitividad, el constructo del ego, el efecto de la ansiedad y el temor a la evaluación negativa.

Palabras clave: biorretroalimentación, personalidad, psicología del deporte, rendimiento deportivo. 


\section{Influencing Factors on Athlete's Motivation}

\section{Abstract}

Nowadays, motivation is one of the areas of greatest interest in high-performance sports practice; in fact, new approaches have emerged and, at the same time, questions have arisen about the reasons that lead the athlete either to increase or decrease her or his performance. The aim of this article is to summarize different factors that influence athletes' motivation. A literature review was carried out by analyzing the literature available in PubMed, Scielo and Dialnet databases that address this topic. With a Price index of $54 \%$, the articles reviewed show that the athlete's state of mind is of great importance, considering the influence it has on the development of self-control and decision-making, which are fundamental elements for the achievement of proposed goals and sporting success. This work highlights the influence that different factors can have on motivation / demotivation and the fulfillment of physical-sports objectives, such as socio-affective bonds, group leadership and cohesion, coach participation, competitiveness, ego construct, effect of anxiety, and fear of negative evaluation.

Keywords: biofeedback, personality, sport psychology, athletic performance. 


\section{Introducción}

En la actualidad, existe una tendencia generalizada a mejorar el rendimiento deportivo a través de metodologías reproducibles y que incluyan, a través de un análisis sistémico, las diferentes variables que influyen en el atleta; de hecho, los planes de trabajo dentro de un programa para optimizar los resultados deportivos buscan integrar aspectos emocionales y sociales por medio de los aportes que puede brindar la psicología deportiva (Cox, 2009). En este sentido, la motivación es una de las variables que ha captado la mayor atención, considerando que es un proceso dinámico y en constante fluctuación que ubica al ser humano en una superficie inestable que depende de una variedad de factores a nivel biológico, emocional, social y cognitivo, para finalmente modular la conducta y el comportamiento. Desde la psicología deportiva, han surgido nuevos planteamientos e interrogantes acerca de las razones que llevan a los deportistas al cumplimiento satisfactorio o al descenso de su rendimiento a nivel motivacional (García, Cervelló, Sánchez y Navas, 2009; Ruiz y Zarauz, 2014; Troncoso, Burgos y López, 2015; Arias, Cardoso, Aguirre y Arenas, 2016).

La motivación se puede definir como el conjunto de factores que llevan al ser humano a comportarse o actuar en una manera de búsqueda de metas o de satisfacción, pudiendo estar influenciada por impulsos psicológicos o por estímulos externos (Mesurado, 2008; Rodríguez, 1991). A menudo se emplean otros términos como necesidades, instintos, motivos, deseos, entre otros, para expresar las acciones del individuo; pero el concepto de motivación abarca estos y es una forma de analizar la toma de decisiones de los seres humanos. Formalmente, según su origen, se distinguen las motivaciones primarias (de supervivencia o viscerales) y las motivaciones secundarias (sociales o psicogénicas) (Reeve, 2010). No obstante, también se pueden clasificar como conscientes o inconscientes, simples o complejas, transitorias o permanentes, y superiores, como lo son los ideales o los modelos existenciales que cada uno asume para su propia realización (Ráez y Herrera, 2009).

Deci y Ryan (1985) señalan que la motivación es una mezcla de motivación extrínseca e intrínseca, encontrada en las necesidades innatas de autodeterminación para la competencia, razón por la cual dicha motivación se ve afectada por diferentes factores que pueden incentivarla o, por el contrario, 
retrasar la posibilidad de alcanzar una meta deseada. De esta manera, no se puede estimar la incidencia de los factores ambientales sin considerar las motivaciones individuales de los deportistas, pues estos pueden mantener un nivel elevado de motivación a pesar de que las circunstancias del entorno no sean las más idóneas (Sánchez y Torregosa, 2005). Por eso, lo más correcto es considerar la motivación como el producto de la interacción de factores internos (personales) y externos (ambientales o situacionales).

Se entiende que la conducta intrínsecamente motivada es aquella que no tiene otro objetivo excepto el propio placer o interés personal. La motivación intrínseca se basa en una serie de necesidades psicológicas, que son responsables de la iniciación, persistencia y resiliencia de la conducta (Reeve, 2010; García-Secades et al., 2014). Este tipo de motivación surge espontáneamente por tendencias internas y psicológicas, sin que haya recompensas externas. Por otro lado, la motivación extrínseca, está relacionada con la perspectiva conductista. Esta corriente no niega que existan causas internas - asimismo influyentes-, pero otorga un papel preponderante a aquellas causas de la conducta que provienen del exterior y que no se encuentran dentro de la persona (e. g., dinero, castigo, espacios y reconocimiento social) (Aguirre y González, 2014). Cabe resaltar la existencia de la no motivación como un estado importante que influye en el rendimiento de los deportistas (Pineda-Espejel, López-Walle y Tomás, 2015).

La mayoría de las investigaciones sobre la motivación en la práctica deportiva se han centrado en el deporte competitivo y de alto rendimiento (Ros-Martínez, Moya-Faz y Garcés, 2013), tanto a nivel individual como por equipos, abarcando no solamente las divisiones mayores sino también categorías infantil y juvenil (e. g., talento y reserva) (Smith y Smoll, 1991; Sampol, Ponseti, Gili, Borras y Vidal, 2005). Además, el análisis de la conducta se ha ido extendiendo a los agentes psicosociales que intervienen en el contexto deportivo (Balaguer, Castillo, Ródenas, Fabra y Duda, 2008; Soriano, Ramis, Cruz y Sousa, 2014), las diferencias sexuales y transculturales (García, Herazo y Tuesca, 2015), la inserción y reajuste social mediado por la práctica recreativa y terapéutica (Alvis y Neira, 2013), así como las aplicaciones del ejercicio físico en la salud y el bienestar. Así, a través de una revisión de la literatura, el presente trabajo pretende resumir la influencia de diferentes factores reportados sobre la motivación de los deportistas. 


\section{Metodología}

Se realizó una revisión narrativa mediante una búsqueda bibliográfica en las bases de datos PubMed, Scielo y Dialnet, con el fin de identificar las publicaciones que evalúan y analizan factores que influyen en el estado motivacional de los deportistas. Los criterios de inclusión para la selección de trabajos fueron los siguientes: i) documentos y artículos publicados desde 2010 hasta 2020; ii) de tipo cualitativo, cuantitativo y de diseños transversales; iii) inclusión de deportistas profesionales, federados, universitarios o escolares de diferentes modalidades deportivas tanto activos como retirados. Las publicaciones de años anteriores constituyen el cuerpo básico del texto. Como medida de obsolescencia se empleó el índice Price.

\section{Resultados y discusión}

El cuerpo de la presente revisión narrativa está compuesto de artículos revisados por pares (índice Price $=54 \%$ ) que cumplieron los criterios de inclusión y que evalúan diferentes aspectos que influyen en el estado de motivación de los deportistas. Las publicaciones revisadas fundamentaron su análisis en la teoría de metas de logro, la teoría de atribuciones causales y la teoría de autodeterminación (Balaguer, Castillo y Duda, 2008; Ráez y Herrera, 2009). La población utilizada en las diferentes investigaciones estuvo conformada por sujetos de ambos sexos, con edades entre 11 y 44 años de edad. Los países con mayor número de investigaciones realizadas en lo que concierne a la temática de este artículo fueron España y Colombia.

Los artículos revisados coinciden en que el estado psicológico motivacional está influenciado por factores internos y externos, que presentan un comportamiento bidireccional y dinámico, que según algunos autores se le conoce como Modelo Jerárquico de Motivación Intrínseca y Extrínseca (HMIEM, por las siglas en inglés de Hierarchical Model of Intrinsic and Extrinsic Motivation) (Sheehan, Herring y Campbell, 2018). Este modelo proporciona un marco para comprender los determinantes y las consecuencias de la motivación a nivel global (personalidad), contextual (dominio de la vida) y situacional (estado) en los individuos, considerando las consecuencias afectivas, cognitivas o conductuales (Vallerand, 2007). La figura 1 representa los factores principales que afectan la motivación del deportista. 
Figura 1. Factores que afecta la motivación del deportista

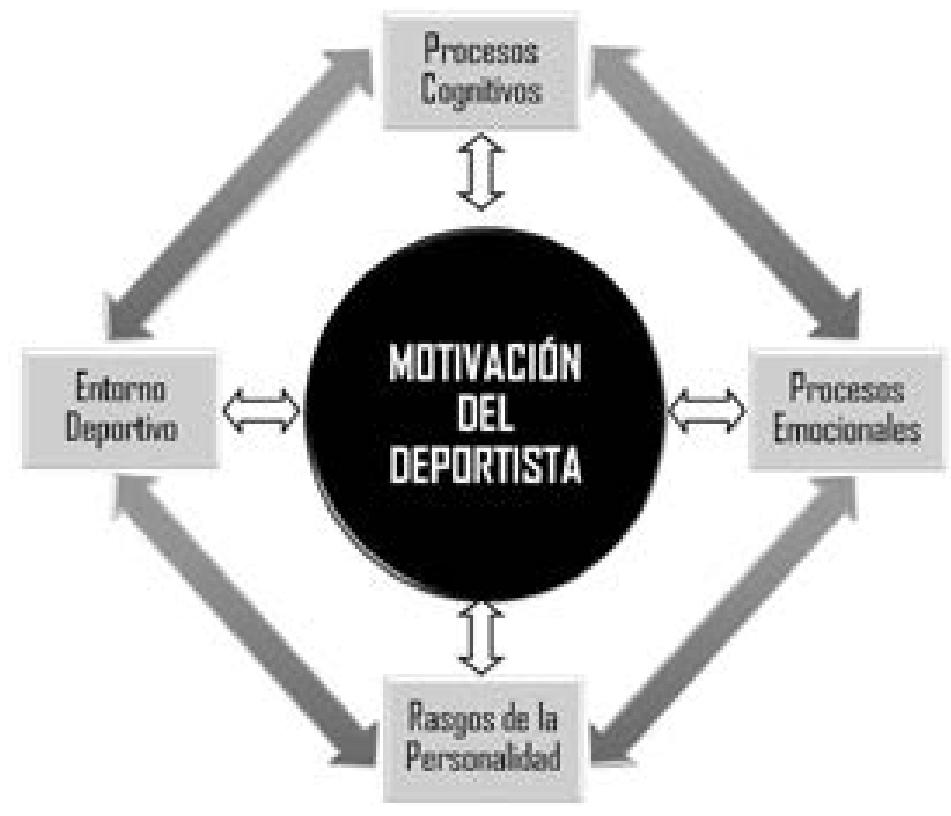

Fuente: elaboración propia.

Los procesos cognitivos (autoconfianza y pensamientos negativos para la toma de decisiones), emocionales (estados de ánimo, vínculos socioafectivos y miedo al fracaso y la evaluación-ansiedad deportiva), rasgos de la personalidad (el constructo del ego, perfil optimista) y del entorno (objetivos físico-deportivos, la participación del entrenador, competitividad, liderazgo y cohesión del grupo) regulan de manera dinámica, y generalmente bidireccional, el estado de motivación del atleta.

Cuando se habla de motivación, las redes amorosas tienen una gran importancia en las relaciones socioafectivas y el rendimiento de los deportistas (Diez, Requena y Zabiaur, 2012), aunque también es importante señalar que los jugadores de alto rendimiento presentan una mayor motivación intrínseca (orientados a la tarea), considerando que se le da más importancia al proceso y se busca el mantenimiento de la práctica deportiva durante gran parte de sus vidas (García y Remor, 2011). De hecho, una alta autoconfianza y 
la búsqueda del mantenimiento de un óptimo acondicionamiento físico para cumplir metas deportivas (Díaz y Mora, 2013) y descanso adecuado (Halson, 2019; Ritland et al., 2019) llegan a tener una relación positiva con el rendimiento deportivo, lo que parece apoyar cierta inclinación hacia la motivación centrada en la tarea por encima de la motivación extrínseca o la relacionada con el ego (Arias et al., 2016).

No obstante, se debe hacer una distinción entre los atletas de deportes de equipo y aquellos de deportes individuales. Por ejemplo, la motivación intrínseca parece repetirse en deportes como voleibol (Reyes, Raimundi y Gómez, 2012) y baloncesto (Sánchez y Ruiz, 2014), pero no parece estar muy presente en deportes individuales como el maratón, donde priman las motivaciones relacionadas con el ego y la competición (Ruiz y Zarauz, 2014), aunque es importante resaltar que los atletas de disciplinas individuales son más propensos a reportar ansiedad y depresión que los atletas de deportes de equipo (Pluhar et al., 2019). A pesar de lo mencionado anteriormente, no es posible realizar una categorización absoluta del tipo de motivación si hablamos de deporte en equipo o individual, teniendo en cuenta que existen prácticas deportivas con un comportamiento híbrido, como se ha reportado en los jugadores de fútbol (García y Remor, 2011). En estos términos, la propuesta para abordar esta clasificación (intrínseca, extrínseca o no motivación) es referirse en términos de predominancia, atendiendo a que todas coexisten en los seres humanos.

Ahora bien, durante la revisión se observó que los investigadores contemporáneos tienen como línea de investigación principal el análisis de la influencia de variables temporales sobre el desarrollo socioafectivo de jugadores profesionales, en donde se pueden encontrar manifestaciones de apego hacia el equipo, los padres y el entrenador (Balaguer et al., 2015), al ser agentes que influyen en el proceso educativo y de formación deportiva. En este sentido, se ha tenido en cuenta la interacción entrenador-atleta para la comprensión de las prácticas de liderazgo, los climas motivacionales y la comunicación entre jugadores y líderes deportivos; dando a entender la importancia del trato democrático por parte del entrenador para un desarrollo motivacional satisfactorio en los deportistas (Troncoso et al., 2015; Fransen, Boen, Vansteenkiste, Mertens y Vande Broek, 2018; Ruiz, Robazza, Tolvanen, Haapanen y Duda, 2019). De hecho, Akyüz, Agar, Akyüz y Dogru (2016) consideran que los factores de motivación externa (i. e., condiciones ambientales, instalaciones deportivas de las escuelas, los medios de comunicación) tienen poca influencia; sin embargo, los entrenadores, 
el entorno amistoso y los profesores de educación física hacen parte de las razones principales por las cuales el atleta elije su rama deportiva.

Se han indagado las características psicológicas en deportes de conjunto en diferentes condiciones socioculturales, destacándose diferencias entre hombres y mujeres; por ejemplo, las características psicológicas en las mujeres abarcan la cohesión del equipo, mayor actividad mental en pro del grupo, aunque mayores niveles de ansiedad deportiva, mientras que los hombres destacan en su control del estrés, influencia sobre el rendimiento pero un mayor miedo al fracaso (Arias et al., 2016). Suponiendo que la preparación psicológica de los deportistas es proporcional a las exigencias propias de cada deporte y la intervención psicológica misma, se han presentado programas de intervención de habilidades psicológicas deportivas que implican la promoción del aprendizaje y modificación de diversas habilidades como la confianza y sentido de la práctica deportiva, además de acompañamiento y mejoramiento en el ámbito personal y académico, demostrándose que la mejora de estas habilidades (autoconfianza, atención, concentración y visualización) influye de manera significativa en el rendimiento deportivo (Reyes et al., 2012). No obstante, si se analizan la relación entre el rasgo de competitividad y el rendimiento deportivo en función del nivel de profesionalización (i. e., categoría), se determina que a mayor profesionalización se observa un mayor rendimiento; de hecho, se observa que la situación ejerce un efecto sobre la conducta, de forma que, si el contexto es de logro, tiende a observarse mayor expresión de la conducta de rendimiento óptimo y de competitividad.

Esto se ha evidenciado al someter a una alta presión a los deportistas para obtener resultados y poder pasar a categorías superiores, ya que se modifica la capacidad de atención, la concentración y la motivación de los jugadores (García y Remor, 2011). Se ha comprobado la relación de predicción entre motivación y estrategias de afrontamiento en futbolistas profesionales y no profesionales, observándose que los primeros muestran más autoconfianza y motivación para superar los obstáculos del contexto deportivo, evidenciando así el uso de estrategias centradas en la emoción para hacer frente a los agentes estresores (Pestillo et al., 2016). También es relevante mencionar que existen factores que facilitan el acceso a las máximas competiciones, refiriéndose a las actividades que llevan los mismos clubes y que permiten ingresar a categorías superiores.

En este sentido, ciertas oportunidades como la formación integral, la modulación de los niveles de presión y la identificación temprana de deportistas 
con potencial generan confianza a los jugadores y facilitan su desarrollo deportivo (Sánchez y Ruiz, 2014). Los propios deportistas consideran que la existencia de oportunidades y de normas federativas favorece el acceso a competiciones de mayor nivel, aunque también se resalta la importancia que adquieren los padres, los familiares y el entrenador sobre la persistencia o el abandono de la práctica deportiva en jóvenes estudiantes; por lo tanto, el núcleo familiar y las personas cercanas al atleta establecen, de cierta manera, los primeros procesos de presión como predictor de la continuidad deportiva en etapas de iniciación (Ramis, Torregrosa, Viladrich y Cruz, 2013). Sánchez Leo, Sánchez, Amado y García (2012) concluyen que existe una asociación entre el compromiso de los jóvenes en edad escolar con la importancia que da el entrenador al esfuerzo y colaboración de cada miembro de un equipo, resaltando de manera interesante que los compañeros de juego no aparecen como predictores de continuidad o cese de la práctica deportiva.

$\mathrm{Al}$ analizar la relación entre el rendimiento deportivo y las orientaciones, los climas motivacionales y las atribuciones realizadas, atendiendo a diferencias entre perfiles de atribuciones inherentes de los deportistas (Cece, Lienhart, Nicaise, Guillet-Descas y Martinent, 2018), se da relevancia al proceso de evaluación y de corroboración de beneficios de los aspectos relacionados con la motivación hacia la tarea. Así, el miedo a la evaluación negativa y los niveles de autoestima/autoconfianza son factores moduladores potenciales del choking (caída del rendimiento deportivo asociado a la presión psicológica), debido a que, si la práctica deportiva se lleva a cabo en condiciones de alta presión psicológica, el miedo a la evaluación y su resultado interfiere de maneja importante en el rendimiento.

García et al. (2009) resaltan cambios significativos en algunas variables de rendimiento deportivo (errores no forzados y puntos conseguidos) en sujetos con miedo a la evaluación durante periodos de alta presión. En contraste, si la práctica deportiva se lleva a cabo en una condición de baja presión, el efecto del miedo a la evaluación negativa resulta ser facilitador del rendimiento deportivo, lo cual constata que la autoestima/autoconfianza favorece en igual medida el rendimiento deportivo, en función de las condiciones de presión. En particular, los jugadores con altos niveles de autoconfianza cometen más errores no forzados durante la condición de baja presión, lo que podría deberse a que estos jugadores participan y se arriesgan más en el juego (Molina, Chorot, Valiente y Sandin, 2014). 
Cabe resaltar el aporte de la mujer en la transformación de los estereotipos socioculturales del deporte, resaltando el contexto colombiano en donde se ha evidenciando un aumento progresivo de la participación femenina e interés en diferentes disciplinas deportivas, incluso en aquellas que eran consideradas solo para hombres (tiro, esgrima, levantamiento de pesas y deportes de combate) (Lucumi, 2012). El modelo de trabajo colaborativo y empático de la naturaleza femenina brinda un punto de vista interesante hacia una motivación intrínseca que se basa en la optimización de procesos. Un estudio de caso interesante fue el realizado por Galatti, Paes, Machado y Montero (2015), quienes analizaron los factores determinantes para el excelente rendimiento que tuvieron las campeonas del mundo de baloncesto. En dicho trabajo se resaltó la supervisión multidisciplinar, las reuniones constantes del equipo (cohesión de grupo), la libertad, la autonomía y la orientación de las jugadoras más veteranas en momentos decisivos.

Estudios previos ya han analizado la relación existente entre cohesión y liderazgo en equipos deportivos (Lozada, Rocha y Castillo, 2012). La falta de expectativas de ser campeonas fue considerado como un punto positivo que evitó presiones y facilitó el resultado alcanzado por estas jugadoras de baloncesto (Galatti et al., 2015). En este sentido, varias publicaciones han resaltado la importancia de la inteligencia emocional, que está relacionada con la capacidad de los atletas para comprender y controlar sus propias emociones y las de otros compañeros, al asociarse positivamente con la motivación deportiva más autodeterminada de los atletas, que refleja el interés y los valores genuinos de una persona en una actividad (Ros-Martínez et al., 2013; Jordalen, Lemyre, Solstad y Ivarsson, 2018; Sukys, Tilindiené, Cesnaitiene y Kreivyte, 2019). Es importante resaltar que todo lo mencionado anteriormente influye en los estados de ánimo de los deportistas en momentos de precompentencia, lo cual ha mostrado afectar el rendimiento (Díaz, Gutiérrez y Hoyos, 2015).

Finalmente, se ha evaluado la influencia del efecto placebo y nocebo en diferentes contextos, con el fin de incrementar el rendimiento deportivo (Beedie, Coleman y Foad, 2007; Hurst, Foad, Coleman y Beedie, 2017). A pesar del potencial, este fenómeno ha constituido una limitación considerando la carencia en la estandarización metodológica que ha hecho frecuente su aplicación como un procedimiento empírico o no intencional que tiene deficiencias en el control de variables. El efecto placebo en la práctica deportiva es concebido como un conjunto heterogéneo de eventos psicobiológicos 
atribuibles a un contexto en donde el deportista, tomando en cuenta sus rasgos de personalidad y motivaciones, interactúa con la figura de activación a través de la ejecución de una intervención (Beedie et al., 2007).

El empleo del placebo puede llevarse a cabo tanto de forma consciente como no consciente, reforzando la intención del deportista para alcanzar sus metas. Para ello, la figura de activación puede auxiliarse con las potencialidades que le proporcionan la sugestión verbal y el priming, principalmente dentro de un ambiente de entrenamiento o competencia que reúna las condiciones óptimas para su utilización (Larquin-Castillo, González y Rodríguez, 2015). Además de la entrevista a profundidad semiestructurada, los instrumentos de evaluación aplicados en las investigaciones analizadas en esta revisión, y que podrían utilizarse dependiendo del contexto en particular que se quiera estudiar, abarcan los siguientes: i) el cuestionario de clima motivacional percibido en el deporte (PMCSQ-2, el cual se encarga de medir la percepción de implicación en la tarea y la percepción del clima de implicación del ego); ii) el cuestionario de orientación al ego y a la tarea en el deporte (TEOSQ); iii) el cuestionario de clima en el deporte (SCQ); iv) el cuestionario de estilo controlador del entrenador (CCBS); v) el cuestionario de características psicológicas relacionadas con el rendimiento deportivo (CPRD); vi) la Causal Dimension Scale-II (CDS-II); vii) el perfil de estados de ánimo (POMS), y viii) el Brief Fear of Negative Evaluation (BFNE). Estos cuestionarios se encuentran dentro de los instrumentos con gran solidez psicométrica para evaluar la motivación, de manera que pueden proporcionar una orientación objetiva a los investigadores y profesionales que deseen proceder a su cuantificación (Clancy, Herring y Campbell, 2017).

\section{Conclusiones}

La motivación es uno de los predictores más importantes de la conducta humana; por lo tanto, es imprescindible tener en cuenta varios aspectos intrínsecos y extrínsecos que intervienen en su desarrollo, con el objetivo de realizar un acercamiento exitoso según las metas deportivas propuestas. El análisis de la literatura revisada permite inferir que tanto los vínculos socioafectivos como la participación del entrenador (liderazgo) influyen profundamente en el estado motivacional de los deportistas. Además, se observó que las personas altamente competitivas tienden a buscar situaciones de desafío y están motivadas 
para obtener éxito en ellas, lo que es un reflejo de la dimensión proactiva de la personalidad que involucra la capacidad para organizar la conducta atendiendo a objetivos y metas. Se confirman diferencias en el comportamiento acorde a las características psicológicas de las disciplinas deportivas, al igual que aspectos relevantes a tener en cuenta que diferencian ambos sexos; no obstante, se requiere más investigación respecto a la influencia de factores extrínsecos sobre la motivación de los deportistas según disciplinas específicas. En conclusión, los factores principales que generan cambios en el estado de motivación de los deportistas son los vínculos socio-afectivos, el liderazgo y la cohesión del grupo, la participación del entrenador, la competitividad, el constructo del ego, el efecto de ansiedad y el temor a la evaluación negativa.

\section{Referencias}

Balaguer, I., Castillo, I., Ródenas, L., Fabra, P. y Duda, J. (2015). Los entrenadores como promotores de la cohesión del equipo. Cuadernos de Psicología del Deporte, 15(1), 233-242. DoI: https://doi.org/10.4321/s1578-84232015000100022

Beedie, C. J., Coleman, D. y Foad, A. (2007). Positive and negative placebo effects resulting from the deceptive administration of an ergogenic aid. International Journal of Sport Nutrition and Exercise Metabolism, 17(3), 259-69. DOI: https://doi.org/10.1123/ijsnem.17.3.259

Cece, V., Lienhart, N., Nicaise, V., Guillet-Descas, E. y Martinent, G. (2018). Longitudinal Sport Motivation Among Young Athletes in Intensive Training Settings: The Role of Basic Psychological Needs Satisfaction and Thwarting in the Profiles of Motivation. Journal of Sport and Exercise Psychology, 40(4), 186-195. Dor: https://doi.org/10.1123/jsep.2017-0195

Clancy, R. B., Herring, M. P. y Campbell, M. J. (2017). Motivation Measures in Sport: A Critical Review and Bibliometric Analysis. Frontiers in psychology, 8, 348. DOI: https://doi.org/10.3389/fpsyg.2017.00348

Cox, R. (2009). Psicología del deporte: concpetos y sus aplicaciones. Buenos Aires: Editorial Médica Panamericana.

Deci, E. y Ryan, R. (1985). Intrinsic motivation and self-determination in human behavior. New York: Springer US.

Díaz, J., Gutiérrez, J. y Hoyos, J. (2015). Estados de ánimo precompetitivos y resultados deportivos en jugadores de Bolos cántabros. Cuadernos de Psicología del Deporte,15(3), 53-64. DoI: https://doi.org/10.4321/s1578-84232015000300005 
Diaz, J. y Mora, J. (2013). Revisión de algunas variables relevantes en el establecimiento de metas deportivas. Anales de Psicología, 29(1), 233-242. doi: https:// doi.org/10.6018/analesps.29.1.137281

Diez, G., Requena, M. y Zabiaur, M. (2012). Influencia del contexto de alto rendimiento deportivo en los vínculos socioafectivos de jugadores de baloncesto. Cuadernos de Psicología del Deporte, 12(1), 93-102. DoI: https://doi. org/10.4321/s1578-84232012000100008

Fransen, K., Boen, F., Vansteenkiste, M., Mertens, N. y Vande Broek, G. (2018). The power of competence support: The impact of coaches and athlete leaders on intrinsic motivation and performance. Scandinavian Journal of Medicine \& Science in Sports, 28(2), 725-745. DOI: https://doi.org/10.1111/sms.12950

Galatti, L., Paes, R., Machado, G. y Montero, A. (2015). Campeonas del mundo de baloncesto: factores determinantes para el rendimiento de excelencia. Cuadernos de psicología del deporte. Cuadernos de Psicología del Deporte, 15(3), 187-192. DOI: https://doi.org/10.4321/s1578-84232015000300020

García, A. y Remor, E. (2011). Motivación de logro, indicadores de competitividad y rendimiento en un equipo de jugadores de futbol de competición varones entre 14 y 24 años. Universitas Pshycologica, 10(2), 477-487. Dor: https://doi. org/10.11144/javeriana.upsy10-2.mlic

García, F., Herazo, Y. y Tuesca, R. (2015). Factores sociodemográficos y motivacionales asociados a la actividad física en estudiantes universitarios. Revista Médica de Chile, 143, 1411-1418. DoI: https://doi.org/10.4067/s0034-98872015001100006

García, T., Cervelló, E., Sánchez P. y Navas, L. (2009). Análisis de las relaciones entre la motivación y las atribuciones causales en jóvenes deportistas. Revista Latinoamericana de Psicología, 42(1), 75-85. Dor: http://dx.doi.org/10.14349/ rlp.v42i1.407

García-Secades, X., Molinero, O., Barquín, R., Salguero, A., De la Vega, R. y Márquez, S. (2014). La resiliencia en el deporte: fundamentos teóricos, instrumentos de evaluación y revisión de la literatura. Cuadernos de Psicología del Deporte, 14(3), 83-98. DOI: https://doi.org/10.4321/s1578-84232014000300010

Halson, S. L. (2019). Sleep monitoring in athletes: motivation, methods, miscalculations and why it matters. Sports Medicine, 49(10), 1487-1497. doi: https://doi. org/10.1007/s40279-019-01119-4

Hurst, P., Foad, A., Coleman, D. y Beedie, C. (2017). Athletes intending to use sports supplements are more likely to respond to a placebo. Medicine and Science in Sports and Exercise, 49(9), 1877-1883. DoI: https://doi.org/10.1249/ mss.0000000000001297 
Jordalen, G., Lemyre, P. N., Solstad, B. E. y Ivarsson, A. (2018). The role of selfcontrol and motivation on exhaustion in youth athletes: a longitudinal perspective. Frontiers in psychology, 9, 2449. DoI: https://doi.org/10.3389/ fpsyg.2018.02449

Larquin-Castillo, M., González, Á. y Rodríguez, B. (2015). Intervenciones placebo para incrementar el rendimiento deportivo: un tema revisitado. Revista Colombiana de Psicología, 24(1), 85-97. DoI: https://doi.org/10.15446/rcp. v24n1.40397

Lozada, E., Rocha, D. y Castillo, L. (2012). Relación entre cohesión y liderazgo en equipos deportivos del departamento de Boyacá-Colombia. Cuadernos de Psicología del Deporte, 12(1), 33-44. DoI: https://doi.org/10.4321/ s1578-84232012000100004

Lucumi, Y. (2012). Aportes de la mujer en la transformación de los estereotipos socio-culturales del deporte colombiano. Revista U.D.C.A Actualidad \& Divulgación Científica, 15(Supl. Olimpismo), 27-35. Recuperado de http:// www.scielo.org.co/pdf/rudca/v15s1/v15s1a05.pdf

Mesurado, B. (2008). Explicaciones psicológicas sobre la motivación y el sustrato neurobiológico que posibilita la misma. Psicologia y Psicopedagogía, 7(19), 1-14. Recuperado de https://racimo.usal.edu.ar/id/eprint/4619

Molina, J., Chorot, P., Valiente, R. y Sandin, B., (2014). Miedo a la evaluación negativa, autoestima y presión psicológica: efectos sobre el rendimiento deportivo en adolescentes. Cuadernos de Psicología del Deporte, 14(3), 57-66. DoI: https://doi.org/10.4321/s1578-84232014000300007

Pestillo, L., Andrade, J., Joao, R., Ferreira, L., Norraila, P. y López, J. (2016). Motivación auto determinada y estrategias de afrontamiento en futbolistas: Un estudio con jugadores en diferentes fases de desarrollo deportivo. Revista de Psicología del Deporte, 25(2), 261-269. Recuperado de https://www.redalyc. org/articulo.oa? $\mathrm{id}=235146515007$

Pineda-Espejel, A., López-Walle, J. y Tomás, I. (2015). Factores situacionales y disposicionales como predictores de la ansiedad y autoconfianza precompetitiva en deportistas universitarios. Cuadernos de Psicología del Deporte, 15(2), 55 70. DOI: https://doi.org/10.4321/s1578-84232015000200007

Pluhar, E., McCracken, C., Griffith, K. L., Christino, M.A., Sugimoto, D. y Meehan, W. P. (2019). Team sport athletes may be less likely to suffer anxiety or depression than individual sport athletes. Journal of Sports Science \& Medicine, 18(3), 490-496. Recuperado de https://www.ncbi.nlm.nih.gov/pmc/articles/ PMC6683619/ 
Ráez, M. y Herrera, D. (2009). Teorías contemporáneas de la motivación: una perspectiva aplicada. Revista de Psicología, 27(2), 311-316. Recuperado de https:// www.redalyc.org/articulo.oa?id=337829513006

Ramis, Y., Torregrosa, M., Viladrich, C. y Cruz, J. (2013). El apoyo a la autonomía generado por entrenadores, compañeros y padres y su efecto sobre la motivación auto determinada de deportistas de iniciación. Anales de Psicología, 29(1), 243-248. DoI: https://doi.org/10.6018/analesps.29.1.124011

Reeve, J. (2010). Motivación y emoción. Ciudad de México: Mc Graw-Hill/ Interamericana.

Reyes, M., Raimundi, M. y Gómez, L. (2012). Programa de entretenimiento en habilidades psicológicas en jugadoras de Voleibol de alto rendimiento. Cuadernos de Psicología del Deporte, 12(1), 9-16. DoI: https://doi.org/10.4321/ s1578-84232012000100001

Ritland, B. M., Simonelli, G., Gentili, R. J., Smith, J. C., He, X., ... Hatfield, B. D. (2019). Effects of sleep extension on cognitive/motor performance and motivation in military tactical athletes. Sleep Medicine, 58, 48-55. Dor: https://doi. org/10.1016/j.sleep.2019.03.013

Rodríguez, R. (1991). Motivación y personalidad. Madrid: Ediciones Déaz de Santos.

Ros-Martínez, A., Moya-Faz, F. y Garcés, E. (2013). Inteligencia emocional y deporte: situación actual del estado de investigación. Cuadernos de Psicología del Deporte, 13(1), 105-112. DoI: https://doi.org/10.4321/ s1578-84232013000100011

Ruiz, F. y Zarauz, A. (2014). Análisis de la motivación en corredores de maratón españoles. Revista Latinoamericana de Psicología, 46(1), 1-11. DoI: https:// doi.org/10.1016/s0120-0534(14)70001-9

Ruiz, M. C., Robazza, C., Tolvanen, A., Haapanen, S. y Duda, J. L. (2019). CoachCreated Motivational Climate and Athletes' Adaptation to Psychological Stress: Temporal Motivation-Emotion Interplay. Frontiers in Psychology, 10, 617. DOI: https://doi.org/10.3389/fpsyg.2019.00617

Sampol, P., Ponseti, X., Gili, M., Borras, P. y Vidal, J. (2005). Motivos para el inicio, mantenimiento y abandono de la práctica deportiva de los preadolescentes de la isla de Mallorca. Apunts. Educación Física y Deportes, 3(81), 5-11.

Sánchez, X. y Torregosa, M. (2005). El papel de los factores psicológicos en la escalada deportiva: un análisis cualitativo. Revista de Psicología del Deporte, 14(2), 177-194. Recuperado de https://www.rpd-online.com/article/viewFile/180/180 
Sánchez, P., Leo, F., Sánchez, D., Amado, D. y García, T. (2012). La influencia del entorno próximo a la persistencia en la práctica de la actividad físico deportiva. Cuadernos de Psicología del Deporte, 12(1), 17-24. Dor: https://doi.org/10.4321/ s1578-84232012000100002

Sánchez, M. y Ruiz, L. (2014). Factores que influyen en el ascenso a la máxima categoría de jugadores de baloncesto. Cuadernos de Psicología del Deporte, 14(3), 67-74. DOI: https://doi.org/10.4321/s1578-84232014000300008

Sheehan, R. B., Herring, M. P. y Campbell, M. J. (2018). Associations between motivation and mental health in sport: a test of the hierarchical model of intrinsic and extrinsic motivation. Frontiers in Psychology, 9, 707. DoI: https:// doi.org/10.3389/fpsyg.2018.00707

Smith, R. y Smoll, F. (1991). Behavioral research and intervention in youth sports. Behaviour Therapy, 22(3), 329-344. DoI: https://doi.org/10.1016/ s0005-7894(05)80370-3

Soriano, G., Ramis, Y., Cruz, J. y Sousa, C. (2014). Un programa de intervención individualizado con entrenadores de fútbol. Cuadernos de Psicología del Deporte, 14(3), 99-106. DOI: https://doi.org/10.4321/s1578-84232014000300011

Sukys, S., Tilindienè, I., Cesnaitiene, V. J. y Kreivyte, R. (2019). Does Emotional Intelligence Predict Athletes' Motivation to Participate in Sports? Perceptual and Motor Skills, 126(2), 305-322. DoI: https://doi.org/10.1177/0031512518825201

Troncoso, S., Burgos, C. y López, J. (2015). Climas motivacionales, liderazgo y cohesión grupal en contexto deportivo universitario. Educación Física y Ciencia, 17(1), 1-12. Recuperado de http://www.memoria.fahce.unlp.edu.ar/art_revistas/pr.6705/pr.6705.pdf

Vallerand, R. J. (2007). A hierarchical model of intrinsic and extrinsic motivation for sport and physical activity. En M. S. Hagger y N. L. D. Chatzisarantis (Eds.), Intrinsic motivation and self-determination in exercise and sport (pp. 255-279). Human Kinetics. 
